\title{
ESTRUCTURA FACTORIAL EXPLORATORIA DE UNA ESCALA DE ACTITUD HACIA GRUPOS CERCANOS A PORTADORES DE VIH / SIDA.
}

\author{
EXPLORATORY FACTORIAL STRUCTURE OF AN ATTITUDE SCALE TOWARDS \\ GROUPS CLOSE TO HIV / AIDS CARRIERS.
}

\author{
Fermín Aguiano-Salazar ${ }^{1}$, Wilfrido Aldana-Balderas², Oscar Valdés-Ambrosio ${ }^{3}$, María \\ de los Ángeles Delgado-Carrillo ${ }^{4}$, Cruz García Lirios ${ }^{5 *}$ \\ ${ }^{[1]}{ }^{[4]}$ Universidad Autónoma de la Ciudad de México (UACM), Ciudad de México - México. \\ [2] [5] Universidad Autónoma del Estado de México (UAEM), Huehuetoca - México. \\ ${ }^{[3]}$ Universidad Nacional Autónoma de México (UNAM), Ciudad de México - México.
}

Recibido mayo de 2018/Received May, 2018

Aceptado agosto de 2018/Accepted August, 2018

\begin{abstract}
RESUMEN
En el marco de las políticas de salud, los procesos psicológicos de identidad y estigma son un binomio que trata de explicar procesos de evaluación de estilos de vida relacionadas con grupos vulnerables. Precisamente, el objetivo del presente trabajo es establecer la validez y confiabilidad de un instrumento que midió ambas dimensiones en una escala de actitudes. Se llevó a cabo un estudio transversal y correlacional a fin de poder establecer las propiedades psicométricas del instrumento con una muestra no probabilística e intencional de 258 estudiantes de una universidad pública. Los resultados muestran ambas dimensiones como factores preponderantes de la actitud, pero su inclusión en el constructo fue asumida como parte de un proceso de activación espontaneo; acciones y riesgos que sólo un grupo cercano a portadores de VIH/SIDA puede llevar a cabo.
\end{abstract}

Palabras Clave: Identidad, estigma, actitud, confiabilidad, validez.

\begin{abstract}
Identity and stigma are a team that tries to explain assessment processes related lifestyles vulnerable groups. The precise purpose of this study is to establish the validity and reliability of an instrument that measured both dimensions on a scale of attitudes. A cross-sectional correlational study was conducted to establish the psychometric properties of the instrument with a nonrandom sample of 258 students from a public university. The results show both dimensions as major factors of attitude, but their inclusion in the construct was taken as part of a process of spontaneous activation; actions and risks that only a nearbearers of HIV/AIDS group can perform.
\end{abstract}

Key Words: Identity, stigma, attitude, reliability, validity.

\section{Introducción}

El objetivo del presente trabajo fue establecer la confiabilidad y la validez de un instrumento que mide actitudes hacia grupos cercanos a portadores del VIH / SIDA en virtud de que los marcos teóricos y conceptuales, así como empíricos advierten la prevalencia de la identidad y el estigma como dos factores determinantes de las diferencias entre grupos cercanos a portadores de VIH / SIDA y grupos expectantes, enjuiciadores

* Autor correspondiente / Correspondig author: garcialirios@yahoo.com 
o estigmatizadores de las familias y amistades de portadores de VIH / SIDA (Bautista, Delgado, García, Valdés, Hernández, Castro, \& Trujillo, 2016).

Sin embargo, la psicología de la salud en general y la psicología de las actitudes en particular, así como las ciencias del comportamiento y de la salud como enfermería, trabajo social o medicina sólo han construido instrumento que miden actitudes para relacionarlos con escalas que miden creencias o conocimientos, así como inventarios que miden el comportamiento sin considerar los criterios de confiabilidad y validez suficientes para llevar a cabo análisis más complejos como los modelos lineales o los modelos estructurales que permiten una explicación más detallada de las relaciones entre las variables cognitivas y conductuales (García, Carreón, Sandoval, Bustos, \& Aguilar, 2016).

No obstante, la literatura sigue creciendo en cuando a la medición unidimensional de las actitudes hacia portadores del VIH / SIDA, hacia la calidad del servicio público de salud, hacia el desempeño de profesionistas de la salud o hacia quienes estigmatizan la enfermedad y sus portadores sin considerar las dimensiones actitudinales establecidas en la literatura especializada como es el caso del tratamiento biomédico, la rehabilitación socioemocional o el apoyo familiar como factores indispensables para un diagnóstico integral del contexto y la situación de quienes portan la enfermedad y quienes los cuidan o interactúan con ellos (Carreón, Bustos, Hernández, Quintero, \& García, 2015).

Por consiguiente, los instrumentos que miden las actitudes hacia el VIH / SIDA no especifican factores e indicadores al medir dimensiones generales sin relación con decisiones o acciones específicas como es el caso del Cuestionario de Sentido de Vida (CSV-25), Escala de Percepción Ontológica (EPO-18), Escala de Afectos Positivos (EAP-21), Escala de Actitud Religiosa (EAR-20) (Meneses, Avellar, Veloso, Nunes, \& Pinheiro, 2015).

Es menester llevar a cabo las pruebas psicológicas relativas a la consistencia y la convergencia de indicadores más específicos con respecto a las dimensiones reportadas en el estado de la cuestión ya que: 1) las escalas que miden dimensiones generales tienden a establecer un factor preponderante que limita la observación de las dimensiones específicas y sus relaciones con otras homologas; 2) los factores generales nulifican la observación de trayectorias de relaciones de dependencia o reflejantes; 3) la observación de la consistencia interna de escalas y su validez de constructo o discriminante con un factor preponderante limitan la construcción de modelos estructurales que requieren factores e indicadores específicos (Lloret, Ferreses, Hernández, \& Thomas, 2014).

La construcción de una escala de actitudes sugiere la revisión de marcos contextuales, teóricos y empíricos para especificar sus dimensiones y las relaciones con indicadores, aunque los parámetros estadísticos determinan la consistencia y la convergencia de variables latentes y manifiestas (Krimberg, Saldanha, \& Neves, 2014).

\section{Salud pública y VIH / SIDA}

El Virus de Inmunodeficiencia Humana (VIH) es un problema de salud pública debido al proceso de incubación y desarrollo. El ciclo del VIH comprende un periodo estándar de 10 años en los que los infectados siguen teniendo estilos de vida riesgosos, ya que no modifican su comportamiento debido a un síntoma que los obligue al autocuidado (Orcasita, Uribe, \& Valderrama, 2012).

El proceso de contagio y reinfección es más probable, ya que desde que la membrana plasmica y el receptor CD4 son infectados, hasta que se procesa el ARN vírico y se reconstruye el nuevo virón, los diagnósticos no son concluyentes y prolifera el contagio del virus en personas con conductas de riesgo (Berenson, Paprocky, Fishman, Bhusman, El-Bassel, \& Downey, 2015).

De este modo, el ciclo del VIH parece evidenciar estilos de vida de riesgo que es posible observar en cualquier parte del mundo y que podrían estar asociados a creencias y actitudes en torno a los portadores, así como a los grupos a los que pertenecen (Orcasita, Cuenca, Garrido, $\&$ Haderlein, 2018).

De esta manera, 30,1 millones de casos son de adultos, 16,8 millones de mujeres y 1,5 millones de niños. África Subsahariana tiene el mayor número de portadores con 22'900,000 mientras que el Norte de África registra 470 mil casos. En América Latina existen 1,5000 mil portadores y en México sólo 200 mil casos.

Ambos aspectos, el proceso de infección y el aumento desproporcionado en las regiones 
parecen indicar que el problema tiene su origen en las conductas de riesgo mismas que al ser influidas por la identidad de grupos portadores y no portadores convierten al VIH en un problema de salud pública relativo al estigma (Guerra \& Gouveia, 2007).

En ciencias de la salud, los grupos cercanos a enfermos son conocidos como soporte social y son factor preponderante en la adherencia al tratamiento, principal variable determinante de la salud hospitalaria (Davis, Shell y King, 2012).
Identidad y estigma son dos procesos psicosociales que para fines del presente trabajo serán entendidos como atribuciones y elecciones sesgadas a favor de un grupo de pertenencia con respecto a otro grupo de referencia (Cañizo \& Salinas, 2010).

Ambas definiciones subyacen de considerar al VIH/SIDA como un problema de salud pública, la cual cobra sentido en un contexto; económico, político, social, asistencial, simbólico, institucional, organizacional, profesional (véase Tabla 1).

Tabla 1. Contexto social de la salud pública

\begin{tabular}{|c|c|c|c|c|}
\hline Contexto & Dimensiones & Factores & Intervenciones & Indicadores \\
\hline Político & Compromiso & $\begin{array}{l}\text { Construcción de } \\
\text { agenda }\end{array}$ & $\begin{array}{l}\text { Establecimiento de } \\
\text { convenios }\end{array}$ & $\begin{array}{l}\text { Porcentajes de acuerdos establecidos } \\
\text { según condicionantes sociales y locales }\end{array}$ \\
\hline Simbólico & $\begin{array}{l}\text { Normas, significados, } \\
\text { ideologias, visiones }\end{array}$ & $\begin{array}{l}\text { Poder, estigma, } \\
\text { discriminación, } \\
\text { influcncia }\end{array}$ & $\begin{array}{l}\text { Mediatización, } \\
\text { encuadre, } \\
\text { sensibilización, } \\
\text { defensoria, } \\
\text { transformación }\end{array}$ & $\begin{array}{l}\text { Porcentajes en tomo a creencias } \\
\text { relacionadas con la acción del otro más } \\
\text { cercano }\end{array}$ \\
\hline Material & Economia, praxis & $\begin{array}{l}\text { Pobreza, } \\
\text { capacidades, } \\
\text { criminalización }\end{array}$ & $\begin{array}{l}\text { Producción, } \\
\text { redistribución, } \\
\text { capacidad, }\end{array}$ & $\begin{array}{l}\text { Porcentajes de exclusión y marginalidad } \\
\text { asistencial }\end{array}$ \\
\hline Relacional & $\begin{array}{l}\text { Intra e inter- } \\
\text { comunidades, entidades, } \\
\text { instancias }\end{array}$ & $\begin{array}{l}\text { Capitales, } \\
\text { participación }\end{array}$ & $\begin{array}{l}\text { educación, iniciativas, } \\
\text { movilización }\end{array}$ & $\begin{array}{l}\text { Porcentajes de organización y } \\
\text { participación consultiva, deliberativa o } \\
\text { consensual }\end{array}$ \\
\hline Institucional & $\begin{array}{l}\text { Estructura, Incidencia, } \\
\text { prevalencia }\end{array}$ & $\begin{array}{l}\text { Vulnerabilidad, } \\
\text { marginalidad, } \\
\text { exclusión }\end{array}$ & $\begin{array}{l}\text { Atención } \\
\text { especializada, } \\
\text { tratamiento } \\
\text { focalirado }\end{array}$ & $\begin{array}{l}\text { Establecimiento de costos y } \\
\text { financiamientos, estructura hospitalaria } \\
\text { e instalaciones modernas, mortalidad, } \\
\text { orfandad y riesgos; porcentaje de casos } \\
\text { en sectores especificos diagnosticados } \\
\text { con procedimientos estandarizados }\end{array}$ \\
\hline Organizacional & $\begin{array}{l}\text { Prestación, capacidad, } \\
\text { calidad }\end{array}$ & $\begin{array}{l}\text { Clima de relaciones } \\
\text { y tareas }\end{array}$ & Reingenieria, sinergia & $\begin{array}{l}\text { Alianzas estratégicas en cuanto a } \\
\text { formación de voluntariado, procesos de } \\
\text { gestión y promoción, Porcentaje de } \\
\text { solicitantes de diagnóstico y } \\
\text { seguimiento de casos, comunicación de } \\
\text { riesgos y promoción de la salud }\end{array}$ \\
\hline Asistencial & $\begin{array}{l}\text { Conocimiento, } \\
\text { comportamiento, } \\
\text { adherencia, } \\
\text { representación, } \\
\text { felicidad, influencia }\end{array}$ & $\begin{array}{l}\text { Promoción, gestićn, } \\
\text { calidad de vida y } \\
\text { bienestar subjetivo }\end{array}$ & $\begin{array}{l}\text { Canalización, } \\
\text { scguimicnto, } \\
\text { mediación, } \\
\text { promoción, gestión }\end{array}$ & $\begin{array}{l}\text { Porcentaje de autocuidado y } \\
\text { afrontamiento en tomo a la } \\
\text { problemática, formación de actitudes } \\
\text { positivas }\end{array}$ \\
\hline
\end{tabular}

Fuente: Mannell, Comish y Rusell (2014)

En el caso de los grupos cercanos a portadores de VIH/SIDA no sólo se les asocia con estilos de riesgo, sino que se les atribuyen identidades de riesgo (Abbasi,Rafique, Aziz, \& Hussai, 2013).
En la construcción del instrumento que mide las actitudes hacia el VIH / SIDA el marco contextual parece indicar que los grupos cercanos a portadores de la enfermedad son responsables de su calidad de vida y bienestar subjetivo sin 
considerar el desgaste emocional, físico o psicológico que supone tal actividad.

\section{Teoría de las Actitudes hacia el VIH / SIDA}

La Figura 1 muestra los marcos teóricos y conceptuales que explican las actitudes hacia el VIH / SIDA son: 1) Teoría de la Acción razonada, 2) Teoría del Comportamiento Planificado; 3) Teoría del Procesamiento Espontáneo, así como sus factores hegemónicos, pero generales en la explicación de una acción específica.

En referencia a las actitudes, la identidad es una consecuencia de una elección deliberada, planificada y sistemática (Summer, 2011). En este sentido, son tres los marcos teóricos actitudinales que explicaría el favoritismo del endogrupo en detrimento del exogrupo conocido como etnocentrismo, aunque un conflicto al interior del grupo de pertenencia generaría un altercentrismo (Chacón, Barrantes, Comerfold, \& McCoy, 2014).

La Teoría de la Acción Razonada (TAR) sostendría que la identidad y el estigma son productos de un procesamiento de información generalizado sobre un grupo de pertenencia en contraste con las atribuciones a un grupo de referencia (Ferragut \& Ortiz, 2013). En este modelo conceptual, las creencias procesan la información circundante, pero son las actitudes quienes determinarán la elección sesgada de un grupo (Uribe \&Orcasita, 2011). Se trata de categorías en las que la información es concentrada para llevar a cabo una acción específica que enaltezca al endogrupo y soslaye al exogrupo (García \& Rodríguez, 2014).

Sin embargo, la acción deliberada resultante, categorizada de información general, no siempre anticipa comportamientos específicos y más bien requerirá de percepciones de control o controles conductuales percibidos (Jouen \& Zielinski, 2013).

Es así como la Teoría del Comportamiento Planificado (TCP) supone que sólo la información delimitada y su procesamiento, tanto de creencias como de percepciones especificarán la información de un modo tal que anticiparían comportamientos puntuales (Klaus, Piñeres, \& Hincapie, 2010).

Figura 1. Teoria de las Actitudes hacia el VIH / SIDA

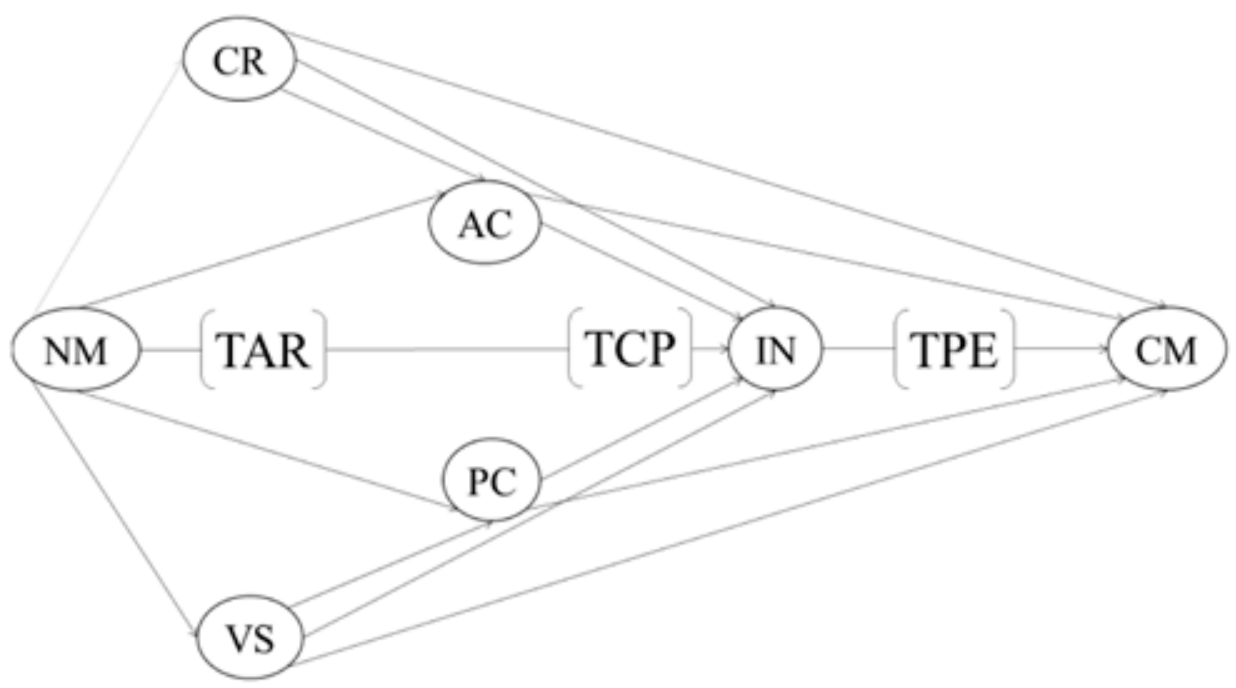

TAR $=$ Teoria de la Acción Razonada, TCP $=$ Teoria del Comportamiento Planificado, TPE

$=$ Teoría del Procesamiento Espontáneo: $\mathrm{NM}=$ Normas, $\mathrm{VS}=$ Valores, $\mathrm{CR}=$ Creencias, $\mathrm{AC}=$ Actitud, $\mathrm{PC}=$ Percepción, $\mathrm{IN}=$ Intención, $\mathrm{CM}=$ Comportamiento.

Fuente: Elaboración propia 
De este modo, la TAR y TCP, identidad y estigma son consecuencias de haber procesado deliberada, planificada y sistemática información concerniente a un grupo muy cercano a un individuo después de haber sido contrasta con información relativa a otros grupos lejanos a ese mismo individuo (Rodríguez, 2013).

No obstante que las actitudes han sido consideradas como asociaciones entre evaluaciones hechas a partir de categorías grupales, la Teoría del Procesamiento Espontáneo (TPE) sostiene que son más bien las actitudes procesos arbitrarios, espontáneos o semiautomáticos (Albacerrin \& Wyer, 2011).

La TPE, a diferencia de la TAR y la TCP que plantean un proceso deliberado, planificado y sistemático, considera que esta información se resguarda en la memoria a largo y corto plazo, así como en su fase procedimental (Mardones \&Guzmán, 2011). De esta forma, la información se almacena y está en un estado de latencia que se activará cuando algún estímulo la recupera y asocia con un comportamiento improvisado (Hughes \& Barnes, 2011).

La identidad y el estigma, desde la óptica de la TPE, son parte del proceso arbitrario, espontáneo o semiautomático que caracteriza a las actitudes (Solat, Velhal, Mahajan, Rao, \& Sharma, 2012). En ese sentido, se advierte que la identidad es una actitud negativa o positiva, a favor o en contra de un grupo y el estigma es una evaluación sesgada de dicho proceso actitudinal e identitario, pero que por algún motivo está latente y no se materializa como un comportamiento hasta que un estímulo reactiva la discrecionalidad del individuo por categorizar a grupos relacionados con portadores de una enfermedad como el VIH/SIDA (Becerra, Chunga, Palomino, Arévalo, Nivin, Portocarrero, Carbajal, Tomás, Caro, Astocaza, Torres, Carbajal, Pinto, Moras, Munayco, \& Gutiérrez, 2012)

De acuerdo con la Teoría del Procesamiento Espontáneo, la memoria no sólo resguarda información concerniente al VIH/SIDA, estilos y conductas de riesgo de grupos vulnerables, sino además tal información es activada espontánea o arbitrariamente para llevar a cabo conductas improvisadas que explicarían el autocuidado. En este sentido, identidad ye estigma serían resultado de la información difundida en los medios, pero indicarían la emergencia de un proceso psicosocial relativo al poder o la influencia social en torno a la sexualidad (Uribe, 2005).

A partir de la TAR, TCP y TPE es posible construir un marco teórico en el que coexisten tanto los procesos deliberados con los espontáneos, planificados con discrecionales, sistemáticos con semiautomáticos (Selesho \& Modise, 2012). En este modelo, la información no fluye de un lado hacia otro o se interconecta de un extremo a otro, sino que está en toda la estructura cognitiva del individuo evidenciando la formación de una red (Villa, 2010).

En tal sentido, la identidad como el estigma no sólo están correlacionadas con las actitudes, sino además son nodos estructurales desde los que la información se resignifica hasta formar nuevos nodos; asociaciones entre categorías y evaluaciones en torno a información concerniente al endogrupo y exogrupo (Méndez, Rojas, \& Moreno, 2012).

Por lo tanto, en la edificación de un instrumento para la medición de las actitudes hacia el VIH / SIDA en general y las actitudes hacia grupos cercanos a portadores del VIH / SIDA en particular es menester considerar: a) Dimensiones razonadas, planificadas y espontáneas, b) indicadores relativos a la aversión y la propensión hacia personas distantes o cercanas a portadores del VIH / SIDA.

\section{Estudios de las Actitudes hacia el VIH / SIDA}

Los estudios psicológicos de las actitudes, la identidad y el estigma (véase Tabla 2) plantean una red cognitiva para explicar las relaciones entre grupos y portadores de VIH/SIDA (Castillo \& Chinchilla, 2011).

Las dimensiones psicosociales de grupos cercanos a portadores de VIH/SIDA: en un contexto de salud pública, en torno al cual se genera información relativa a estilos de vida, conductas de riesgo, violencia y explotación sexual

La identidad y el estigma reportados en el estado del conocimiento respecto a grupos cercanos a portadores de VIH/SIDA: no sólo serían factores preponderantes en su estudio, sino además serían efectos psicosociales de la información relativa a estilos de vida y conductas de riesgo, violencia y explotación sexual diseminados en las percepciones, creencias, actitudes y discursos de grupos con los que familiares y amigos de portadores de VIH/SIDA interactúan. En este sentido, tanto la 
Tabla 2. Estado del conocimiento en torno al VIH/SIDA

\begin{tabular}{|c|c|c|}
\hline Ano & whor & Resultado \\
\hline 2009 & $\begin{array}{l}\text { Chivez, } \\
\text { Petrzelova y } \\
\text { Zapata }\end{array}$ & $\begin{array}{l}\text { El } 70 \% \text { recibió información de sus familiares, el } 97 \% \text { conoció los preservativos, el } 89 \% \text { tenia información } \\
\text { sobre enfernedades de transmisión sexual, el } 51 \% \text { consideró a las relaciones homosexuales como anormales, } \\
\text { el } 41 \% \text { estableció la mayoria de edad como ideal para iniciar una vida sexual, el } 18 \% \text { manifestó que iniciaria } \\
\text { sus relaciones sexuales después del matrimonio, pero el } 18 \% \text { tuvo su primer relación a los } 16 \text { afos, el } 25 \% \\
\text { reconoció haber sido presionado por sus amistadas a tener relaciones sexuales, el } 31 \% \text { declaró que sus padres } \\
\text { verian mla el que tuviera relaciones sexuales, el } 49 \% \text { senaló que respetarian las reglas sobre sexualidad de } \\
\text { sus padres, el } 38 \% \text { consideró que podia quedar embarazada en su primera relación sexual, } 18 \% \text { tuvo } \\
\text { relaciones sexuales bajo los efectos del alcohol. }\end{array}$ \\
\hline 2010 & $\begin{array}{l}\text { Klaus, Pincres e } \\
\text { Hincapie }\end{array}$ & $\begin{array}{l}\text { El objetivo de las investigaciones fue establecer los puntos clave de sa vida interior. En este sentido, las } \\
\text { victimas se consideran en un estado de "pecado" y no de explotación o indefensión. }\end{array}$ \\
\hline 2011 & Aramayo & $\begin{array}{l}\text { Los abusos sexuales en la primera infancia incidieron en la sexualidad adulta. La réplica de las experiencias } \\
\text { sexuales del puso en el presente. La relación entre agresores y viectimas fue explicada a partir de las } \\
\text { experiencias sexuales del pasado. Mediante un inventario de experiencias de agresión sexual estableció un } \\
\text { vinculo con las experiencias sexuales actuales }\end{array}$ \\
\hline 2011 & $\begin{array}{l}\text { Castillo y } \\
\text { Chinchilla }\end{array}$ & $\begin{array}{l}\text { La mayoria de las victimas son ninas o adolescentes entre los } 14 \text { y } 17 \text { antos, alrededor del } 35 \% \text { han sido } \\
\text { abusadas sexualmente y } 40 \% \text { son madres. }\end{array}$ \\
\hline 2011 & $\begin{array}{l}\text { Mardones y } \\
\text { Gurmán }\end{array}$ & $\begin{array}{l}\text { La explotación sexual comercial está relacionada con el consumo de đrogas, negligencia familiar, trastomos } \\
\text { psiquiátricos, deserción escolar y pobreza. La adherencia al tratamiento es menor a to esperado ya que la } \\
\text { victima reincide. }\end{array}$ \\
\hline 2011 & Petracei & $\begin{array}{l}\text { En los casos opuestos, el coito intemuptus es la práctica sexual más prevaleciente en hombres eon un } \\
\text { compromiso afectivo significativo. Relacionó con una red de creencias acerca de invulnerabilidad de los } \\
\text { jóvenes con respecto al contagio del VIH o el no desarrollo del SIDA. }\end{array}$ \\
\hline 2011 & Rivera & $\begin{array}{l}\text { Encontró que las vietimas no siempre se consideran explotadas y más bien son sus creencias religiosas en } \\
\text { torno al pecado las que inciden en sus comportamientos sexuales. Los estudios sobre la explotación sexual } \\
\text { comercial tienden a centrar el problena como trata de blancas, aunque justifican la idea de trafico de } \\
\text { personas, no sustentan las condiciones de explotación o esclavitud sexual ya que las entrevistas con las que } \\
\text { ejemplifican los casos las victimas enfatizan su voluntad de prostituirse para pagar sus deudas o apremios. }\end{array}$ \\
\hline 2011 & rano & $\begin{array}{l}\text { Hombres y mujeres evalóan maternal o paternalmente a su pareja según sea el caso. Una evaluación positiva } \\
\text { implica decisiones compartidas. En cambio, una autoevaluación negativa determina una decisión delegada a } \\
\text { la pareja. Adviente que cuando las parejas establecen una red comunicativa, sus decisiones se llevan a cabo } \\
\text { por consenso, pero cuando sólo se etablece una comunicación unidireceional, los hombres delegan en la } \\
\text { mujer la responsabilidad de la anticoncepción. }\end{array}$ \\
\hline 2011 & $\begin{array}{l}\text { Silva, Ashton y } \\
\text { MeNeil }\end{array}$ & $\begin{array}{l}\text { En general una relación percibida como externa a los intereses y propósitos personales incrementa las } \\
\text { posibilidades de delegar la decisión en la mujer }\end{array}$ \\
\hline 2011 & Unibe y Orcasita & $\begin{array}{l}76 \% \text { declaró haber recibido información sobre el VIH / SIDA, } 41 \% \text { utilizó la televisión para enterarse, } 32 \% \\
\text { reviso articulos de revistas, } 17.6 \% \text { utilizó folletos. Respecto al nivel de fobia } 85 \% \text { no le preocupa contraer el } \\
\text { VIH, pero el mismo porcentaje evita ver programas sobre el tema. } 82.4 \% \text { cree que os cero positivo, con el } \\
\text { mismo porcentaje declararon que podrian morir a causa del SIDA. }\end{array}$ \\
\hline 2012 & Becerra et al.s. & $\begin{array}{l}86.5 \% \text { manifestó un conocimiento aceptable sobre VIH, } 35.2 \% \text { sétaló que podria infectarse por compartir } \\
\text { utensilios con portadores. Los conocimientos y las actitudes se asociaron positivamente. }\end{array}$ \\
\hline 2012 & $\begin{array}{l}\text { Davis, Shell y } \\
\text { King }\end{array}$ & $\begin{array}{l}33 \% \text { de los técnicos atendian pacientes infectados por VIH, el } 86 \% \text { se sentia incómodo con la situación ya } \\
\text { que el } 44 \% \text { no deseaba promover antivirales. }\end{array}$ \\
\hline
\end{tabular}




\begin{tabular}{|c|c|c|}
\hline & Moreno & comercial. \\
\hline 2012 & $\begin{array}{l}\text { Selesho y } \\
\text { Modise }\end{array}$ & $\begin{array}{l}\text { Los estilos de enseflanza influyeron sobre las habilidades y las responsabilidades de los estudiantes. La } \\
\text { preveneión del VIH / SIDA fue posible a partir de la implementación de habilidades sociales. }\end{array}$ \\
\hline 2012 & Solat et lal., & $\begin{array}{l}52.9 \% \text { estuvo consciente de los riesgos de contagio, } 39.9 \% \text { conocia las medidas de prevención, } 43.8 \% \text { nunca } \\
\text { habia usado el condón, y } 17.2 \% \text { tenía conocimiento de su uso correcto }\end{array}$ \\
\hline 2013 & $\begin{array}{l}\text { Abbasi, Rafique, } \\
\text { Aziz\&Hussai }\end{array}$ & $\begin{array}{l}97.9 \% \text { de los entrevistados declararon conocer el proceso de infección. } 94 \% \text { consideraron al virus como } \\
\text { mortal, } 81.6 \% \text { senalan que la infección es una tragedia personal, } 66 \% \text { senalaron que no existe vacuna alguna, }\end{array}$ \\
\hline 2013 & Cobos et al., & $\begin{array}{l}\text { Los grupos manifestaron un bajo nivel de conocimientos respecto a infecciones y enfermedades de } \\
\text { transmisión sexual, asi como la percepción de estigmas en torno al VIH y sus portadores. A partir de tales } \\
\text { hallazgos se considera a la discriminación como la problemática eentral de las comunidades chontales. }\end{array}$ \\
\hline 2013 & Garcia & $\begin{array}{l}\text { Demostró en un grupo de profesionales de la salud estigmatizaron la atención a familiares y portadores de } \\
\text { VIH/SIDA como una minoria vulnerable al que se le debiera de atender de un modo diferente a los demás } \\
\text { grupos de familiares y enfermos }\end{array}$ \\
\hline 2013 & Garcia et al., & $\begin{array}{l}\text { La norma grupal incidió sobre la actitud hacia portadores de VIH }(\beta=, 70) \text {. La relación indirecta entre sexo y } \\
\text { actitud a través de la norma }(\beta=-.47) \text {. }\end{array}$ \\
\hline 2013 & Giraldo & $\begin{array}{l}\text { Delimitar los conceptos de juventud y sexualidad a dimensiones comerciales soslaya factores relativos a la } \\
\text { expresividad. Encontró en la expresividad de la enfermedad una estructura de red asociada a la } \\
\text { responsabilidad social y la solidaridad con portadores en fase terminal. }\end{array}$ \\
\hline 2013 & Hernaindez & $\begin{array}{l}\text { Las mujeres migrantes son discriminadas por ser extranjeras y por su género, ambas segregaciones las hacen } \\
\text { vulnerables a la trata de personas, El traficante utiliza a la familia de las victimas para establecer una relación } \\
\text { de dominio. Descubrió que cuando un grupo es explotado por proxenetas, éstos últimos establecen discursos } \\
\text { que legitiman la superioridad de los residentes u oriundos con respecto a migrantes. }\end{array}$ \\
\hline 2013 & $\begin{array}{l}\text { Hurtado, } \\
\text { Avandaño y } \\
\text { Moreno }\end{array}$ & $\begin{array}{l}\text { Las amistades y companeros de los adolescentes tuvieron una mayor influencia que los padres respecto a la } \\
\text { sexualidad; las preferencias, las experiencias y los estigmas al momento de llevar a cabo un encuentro sexual } \\
\text { o planificar una primera relación. Sostienen que los adolescentes que inician sus relaciones sexuales están } \\
\text { más influidos por sus pares que por sus padres, profesores, grupos sociales o redes digitales. Es decir, la } \\
\text { identidad parece ser un proceso que explicita no sólo la preferencia sexual, sino además la práctica y la } \\
\text { frecuencia de la misma al momento de improvisar o planificar un acto sexual. }\end{array}$ \\
\hline 2013 & $\begin{array}{c}\text { Jouen y } \\
\text { Ziiclinski }\end{array}$ & $\begin{array}{l}\text { El } 57 \% \text { de los turistas fueron curopeos, el } 29 \% \text { declaró prevenir la explotación sexual debido a sus valores } \\
\text { sociales, el } 44 \% \text { utilizaria la denuncia anónima como instrumento de prevención. Sin embargo, el } 48 \% \text { no } \\
\text { conoce las normas punitivas de la explotación sexual. }\end{array}$ \\
\hline 2013 & Mendez & $\begin{array}{l}\text { Evidenció una red de simbolos determinante del reclutamiento de victimas de explotación sexual que } \\
\text { desarrollaron comportamientos de minusvaloración, riesgo y reincidencia. El modo de reclutamiento fue una } \\
\text { oferta laboral. A menudo un familiar o amigo cercano en quien confia la víctima es utilizado para el } \\
\text { reclutamiento. Las victimas fueron trasladadas en transporte publico. el temor a represalias con la victima o } \\
\text { sus familiares inhäbió el escape. El sistema judicial es percibido como corrupto y colidido con los traficantes. } \\
\text { La exclusión social fue asumida como un castigo de la comunidad ante la situación de explotación sexual } \\
\text { comercial. Las experiencias de explotación son asumidas como una motivación para superarse. }\end{array}$ \\
\hline 2013 & Petro & $\begin{array}{l}23.3 \% \text { de los profesores declararon que los estudiantes están informados del impacto de las relaciones } \\
\text { sexuales en su vida académica, } 20 \% \text { señalan que los estudiantes son responsables de su sexualidad, } 33.3 \% \\
\text { manifestaron que la etapa sexual de los estudiantes supone una práctiea temporal, } 53.3 \% \text { advirtieron que los } \\
\text { estudiantes ocupan sa tiempo en relaciones sexuales mis que en su vida academica, } 70 \% \text { consideró que la } \\
\text { educación sexual es determinante de los estilos de vida estudiantiles. Considera que la red cognitiva es un } \\
\text { proceso de responsabilidad social en la que los portadores no sólo evidencian un autocuidado, sino además } \\
\text { diseminan sus experiencias a fin de prevenir reinfecciones o contagios que deriven en un problema de salud } \\
\text { pública }\end{array}$ \\
\hline
\end{tabular}




\begin{tabular}{|c|c|c|}
\hline 2014 & Afanador & $\begin{array}{l}\text { Encontró que la sexualidad es considerada un tabú por los padres al momento de platicar cón sus hijos. Sólo } \\
\text { en los casos en los que la madre tenia una profesión su visión de género predominaba sobre la información } \\
\text { circundante en los medios que asociaron estilos de vida y conductas de riesgo a grupos bomosexuales. } \\
\text { Advirtió un sesgo relativo a la identidad de género al momento de la plática sexual entre padres e hijos y } \\
\text { concluyó que no sólo el tema sexual era un tabú, sino que además estaba orientado por una visión de género } \\
\text { femenina más que una identidad de género masculina. }\end{array}$ \\
\hline 2014 & Chacón et al., & $\begin{array}{l}\text { A pesar de haber encontrado un nivel alto de conocimientos en torno al VIH / SIDA los encuestados al } \\
\text { consumir alcohol, mariguana, crack y cocaína llevaron a cabo prácticas de riesgo que contradice el supuesto } \\
\text { según el cual un nivel suficiente de información conducinia a la práctica sexual segura. }\end{array}$ \\
\hline 2014 & $\begin{array}{l}\text { García y } \\
\text { Rodriguez }\end{array}$ & $\begin{array}{l}\text { Encontraron diferencias significativas entre hombres y mujeres con respecto a su condición de infectados por } \\
\text { estado civil }[\mathrm{U}=383,500 ; \mathrm{p}=0,001] \text {, empleo [483,500; } \mathrm{p}=0,024] \text {, modo de infeceión }[\mathrm{U}=380,000 ; \mathrm{p}= \\
0,000] \text {. uso de drogas [U }=560,000 ; p=0,033] \text {, tipo de drogas }[\mathrm{U}=562,000 ; \mathrm{p}=0,037] \text {. consumo de } \\
\text { alcohol }[\mathrm{U}=518,000 ; \mathrm{p}=0,035] \text {, apoyo familiar }[\mathrm{U}=304,000 ; \mathrm{p}=000] \text { y relaciones sexuales }[\mathrm{U}=550,500 \text {; } \\
p=0,038]\end{array}$ \\
\hline 2014 & $\begin{array}{l}\text { Mannell, } \\
\text { Comishy } \\
\text { Russell }\end{array}$ & $\begin{array}{l}\text { Levaron a cabo una revisión de las intervenciones y los resultados de seis organizaciones intemacionales en } \\
\text { las que encontraron un énfasis en los casos individuales tanto en su aspecto clínico como conductual, } \\
\text { soslayando la complejidad social de la enfermedad; el estigma en tomo a la identidad de género, la } \\
\text { sensibilización politica del VIH y su inserción en la agenda civil como tema de seguridad y salud pública. }\end{array}$ \\
\hline
\end{tabular}

\section{Fuente: Elaboración propia}

identidad como el estigma son dos nodos representativos y discursivos a partir de los cuales se procesa la información concerniente a los riesgos de salud pública. Es decir, la información atribuida a grupos vulnerables al VIH/SIDA justifica y legitima la división sexual entre aquellos grupos que deliberan, planifican y sistematizan sus coitos ante los grupos que arbitraria e improvisadamente tienen encuentros sexuales.

La identidad de género estaría vinculada con el estigma, ya que se trata de dos nodos informativos que si bien fueron influenciados por medios de comunicación orientan la formación de actitudes hacia la elección y preferencia de un grupo heterosexual con respecto a un grupo homosexual. La identidad no sólo es evaluada por los encuestados, sino además está vinculada con asociaciones que ubican a grupos vulnerables en prácticas de riesgo y explotación sexual. Los jóvenes no sólo distinguen su grupo de pertenencia al grupo de referencia en cuestión (familiares y amigos de VIH/SIDA), sino además advierten una tendencia de atributos que los hace evaluar a esos grupos como diferentes al asociarles estilos de vida y conductas de riesgo propios de un sector vulnerable a la explotación sexual.

La identidad y el estigma son nodos de atribución de información concerniente a estilos de vida y conductas de riesgo en un entorno de explotación sexual, pero es evidente que tanto la identidad como el estigma son componentes de un constructo relativo a las actitudes hacia grupos relacionados con portadores de VIH/SIDA. Es decir, la identidad y el estigma son nodos en donde se concentra o genera la información, pero su importancia radica en observar la relación entre grupos vulnerables, marginados o excluidos en torno a portadores del VIH/SIDA ya que estos son parte de un soporte social que determina en buena medida la adherencia al tratamiento.

A partir de la revisión de los hallazgos reportadores en la literatura consultada es posible advertir que los grupos cercanos a portadores del $\mathrm{VIH} /$ SIDA parecen tener una mayor injerencia en la toma de decisiones sólo si el portador considera que la relación con ese grupo es significativa y con un alto compromiso, o bien, si la aversión y la propensión hacia estos grupos está definida y no hay ambivalencia, entonces la actitud será orientada por indicadores presentes más que del pasado o del futuro como es el caso del apego o desapego de una relación interpersonal.

En síntesis, las relaciones contextuales, teóricas y empíricas esgrimidas en la literatura con respecto a las relaciones a observar en las actitudes de grupos cercanos portadores de VIH / SIDA sugieren diferencias significativas entre grupos lejanos o cercanos a personas enfermas. Es decir, un mayor distanciamiento con el fenómeno sugiere una actitud negativa hacia familiares y amistades de enfermos terminales. Es el caso de profesionistas del trabajo social que llevan a cabo 
trámites o el seguimiento administrativo de los casos.

En contraste, una mayor proximidad a los casos como es el caso de la mediación de conflictos denota una mayor preocupación no sólo por la salud y el bienestar del portador de la enfermedad sino, además, sugiere una identificación con los familiares de los enfermos.

Por lo tanto, el instrumento a construir deberá considerar: 1) el contexto de la calidad de los servicios médicos públicos; 2) la teoría de las actitudes en procesos deliberados, planificados y sistemáticos, pero también espontáneos, heurísticos e improvisados; 3) los hallazgos relativos a bajas correlaciones entre factores e indicadores.

¿Cuáles son las dimensiones de las actitudes hacia grupos cercanos a portadores de VIH / SIDA considerando la identidad del grupo y el estigma hacia ese grupo por parte de futuros profesionistas de la salud?

Hipótesis nula: existirán diferencias significativas entre las dimensiones de identidad y estigma establecidas en la revisión contextual, teórica y empírica con respecto a las dimensiones observadas en el estudio.

Hipótesis alterna: No existirán diferencias significativas entre las dimensiones de identidad y estigma revisadas en el marco contextual, teórico y empírico con respecto a las relaciones observadas entre factores e indicadores del estudio con practicantes de la salud pública.

\section{Método}

Se llevó a cabo un estudio exploratorio, transversal y correlacional. Se realizó una selección no probabilística de 258 estudiantes de una universidad pública del Estado de México. El criterio de elección fue tener un conocimiento suficiente del VIH/SIDA y haber interactuado con portadores, sus familiares o amistades.

Sexo. El $49 \%$ de los encuestados fueron mujeres $(\mathrm{M}=339,45$ dólares de ingreso mensual y $\mathrm{DE}=21,37$ dólares), el $48 \%$ fueron hombre (M $=384,58$ dólares y $\mathrm{DE}=19,36$ dólares) y el 3\% no contestó

Edad. El 51\% tiene entre 22 y 29 años $(\mathrm{M}=$ 326,38 dólares de ingreso mensual y $\mathrm{DE}=21,25$ ), el $37 \%$ tiene entre 18 y 22 años $(M=273,29$ y $\mathrm{DE}=18,0)$, el $9 \%$ tienen menos de 18 años $(\mathrm{M}=$ $220,13$ y DE $=10,6)$, el $3 \%$ no contestó
Grupo. El 63\% declaró que no pertenecía a algún grupo en especial $(\mathrm{M}=257,27$ dólares de ingreso mensual y DE $=19,08)$, el 34\% señaló que si pertenecía a un grupo en particular $(\mathrm{M}=$ $345,24$ y DE $=17,20)$, el $3 \%$ no contestó

Preferencias. El 82\% señaló que es de preferencia heterosexual $(\mathrm{M}=259,40$ dólares de ingreso mensual y $\mathrm{DE}=37,29)$, el $15 \%$ se declaró homosexual $(\mathrm{M}=301,27$ y $\mathrm{DE}=18,79)$, el $3 \%$ no contestó

Coito. El $71 \%$ declaró que tuvo relaciones sexuales con personas de diferente sexo antes de los 18 años ( $M=241,28$ dólares de ingreso mensual y $\mathrm{DE}=17,29)$, el $20 \%$ no ha tenido relaciones sexuales $(M=215,01$ y $\mathrm{DE}=18,20)$, el $6 \%$ señaló que tuvo relaciones sexuales con personas del mismo sexo $(\mathrm{M}=253,48$ y $\mathrm{DE}=15,08)$

Fuentes. El $71 \%$ dijo que se enteró del VIH/SIDA en la televisión ( $\mathrm{M}=267,28$ dólares de ingreso mensual y $\mathrm{DE}=17,68)$, el $20 \%$ señaló que se enteró en la escuela $(\mathrm{M}=283,49$ y $\mathrm{DE}=$ $18,39)$, el $6 \%$ declaró que no sabía en qué consistía la enfermedad de transmisión sexual $(\mathrm{M}=254,71$ y $\mathrm{DE}=18,29$ )

Se utilizó la Escala de Actitudes hacia el VIH/SIDA de García (2013), la cual incluye 26 aseveraciones en torno a disposiciones negativas o positivas, favorables o desfavorables hacia grupos cercanos a portadores de VIH/SIDA y sus estilos de vida, conductas de riesgos y vulnerabilidad a la explotación sexual. Cada aseveración incluye cuatro opciones de respuesta que van desde 0 = "nada probable" hasta $3=$ muy probable. $\mathrm{La}$ dimensión de identidad mide las actitudes hacia un grupo cercano a portadores de VIH/SIDA en comparación a grupos que se consideran distantes y alcanzo una confiabilidad (alfa $=0,718$ ) superior a la mínima indispensable. La dimensión de estigma mide las actitudes hacía los estilos de vida y conductas de riesgo, así como la vulnerabilidad a la explotación sexual asociada con grupos cercanos a portadores de VIH/SIDA con respecto a grupos distantes alcanzando una confiabilidad (alfa $=$ $0,702)$ superior a la requerida. La escala general tuvo una consistencia interna (alfa $=0,780$ ) superior a las subescalas de identidad y estigma.

Se ajustó el instrumento original al ámbito universitario considerando los hallazgos reportados en el estado del conocimiento y el contexto de salud pública. Se encuestaron a los estudiantes en el vestíbulo de los servicios médicos y orientación 
sexual previo consentimiento informado y advertencia de que los resultados del estudio no afectarían su estatus académico. Los datos se procesaron en el SPSS versión 20 y AMOS versión 4,0. de dependencia entre los dos factores y sus indicadores con la prueba de correlación de Pearson y de covarianzas (véase Tabla 4).

Tabla 4. Correlaciones y covarianzas entre factores

\begin{tabular}{ccccc}
\hline & $\boldsymbol{F 1}$ & $\boldsymbol{F 2}$ & $\boldsymbol{F 1}$ & $\boldsymbol{F 2}$ \\
\hline $\boldsymbol{F 1}$ & 1,000 & & 1,897 & \\
\hline $\boldsymbol{F 2}$ &, $437^{* * *}$ & 1,000 &, 615 & 1,978
\end{tabular}

\begin{abstract}
F1 = Actitud hacia la identidad de grupos cercanos a portadores VIH / SIDA, F2 = Actitud hacia el estigma de grupos cercanos a portadores de VIH / SIDA: * $\mathrm{p}<, 01 ; * * \mathrm{p}<, 001 ; * * *$ $\mathrm{p}, 001$
\end{abstract}

Fuente: Elaborada con los datos del estudio

Se estimaron medidas de tendencia central y medidas de dispersión para los datos descriptivos del instrumento y coeficientes alfa para la confiabilidad, así como valores de análisis factorial exploratorio de componentes principales con rotación varimax, adecuación y esfericidad. Los valores superiores a 0,70 fueron considerados como evidencia de consistencia interna y los valores KMO superiores a 0,600 fueron asumidos como evidencia de adecuación con niveles significativos menores a 0,05 se consideraron caso de esfericidad. Los pesos factoriales superiores a 0,300 se asumieron como evidencia de validez de constructo.

\section{Resultados}

La Tabla 3 muestra valores superiores a 0,70 para la escala general (alfa de, 785 ) y para los ítems que se midieron por dos subescalas relativas la identidad (alfa de ,780) y el estigma (alfa de ,787) como factores preponderantes de la actitud hacia el VIH/SIDA: la cual se estableció con pesos factoriales superiores a 0,300 mismos que explicaron el $43 \%$ y el $57 \%$ de la varianza total.

La consistencia interna y la validez de constructo señalan que el instrumento puede ser replicado en otros contextos con otras muestras, pero éstas últimas deberán ser estudiantes universitarios ya que la selección de la muestra no fue probabilística.

Una vez establecidos los dos factores, se procedió a contrastar las trayectorias de relaciones
Es posible observar una baja correlación entre los factores lo cual sugiere un factor común y hegemónico, pero una alta covarianza que supone la inclusión de un tercer factor no incluido en el estudio. Se estimó un modelo estructural para establecer al factor preponderante o el aspecto bidimensional de las actitudes hacia grupos cercanos a portadores del VIH / SIDA (véase Figura 2).

Los parámetros de ajuste y residuales $\left\lceil\mathrm{X}^{2}\right.$ $=342,12(56 \mathrm{gl}) \mathrm{p}=, 009 ; \mathrm{CFI}=, 990 ; \mathrm{GFI}=, 0997$; RMSEA $=, 009$ J sugieren el no rechazo de la hipótesis nula relativa al ajuste de las dimensiones de identidad y estigma establecidas en la revisión de la literatura con respecto a las relaciones entre estos factores observadas en el presente trabajo.

\section{Discusión}

El presente estudio ha establecido la validez y confiabilidad de un instrumento que mide la actitud hacia la identidad y el estigma de grupos cercanos a portadores de VIH/SIDA, aunque el tipo de estudio no experimental, el tipo de muestreo no probabilístico y el tipo de análisis no confirmatorio limitan los resultados al escenario de la investigación.

La consistencia interna del instrumento y de las dos subescalas alcanzaron valores superiores a los reportados por García (2013), pero inferiores a otros instrumentos que midieron las actitudes hacia el VIH / SIDA como dimensiones de la calidad de vida y el bienestar subjetivo de Villegas, Rosas y 
Tabla 3. Propiedades psicométricas del instrumento

\begin{tabular}{llllllll}
\hline R & $\boldsymbol{M}$ & $\boldsymbol{D}$ & $\mathbf{S}$ & $\mathbf{C}$ & $\mathbf{A}$ & $\mathbf{F 1}$ & $\mathbf{F 2}$ \\
\hline R1 & 2,48 & 0,17 & 1,40 & 1,21 & 0,713 & 0,482 & \\
\hline R2 & 2,30 & 0,13 & 1,50 & 1,34 & 0,729 & 0,493 & \\
\hline R3 & 2,94 & 0,18 & 1,30 & 1,46 & 0,794 & 0,493 & \\
\hline R4 & 2,60 & 0,09 & 1,96 & 1,48 & 0,739 & 0,491 & \\
\hline R5 & 2,64 & 0,11 & 1,83 & 1,30 & 0,712 & 0,402 & \\
\hline R6 & 2,83 & 0,10 & 1,40 & 1,28 & 0,739 & 0,536 & \\
\hline R7 & 2,49 & 0,13 & 1,30 & 1,32 & 0,725 & 0,406 & \\
\hline R8 & 2,83 & 0,15 & 1,07 & 1,45 & 0,739 & 0,514 & \\
\hline R9 & 2,96 & 0,94 & 1,85 & 1,65 & 0,748 & 0,578 & \\
\hline R10 & 2,77 & 0,85 & 1,36 & 1,76 & 0,745 & 0,351 & \\
\hline R11 & 2,49 & 0,71 & 1,57 & 1,54 & 0,741 & 0,362 & \\
\hline R12 & 2,61 & 0,39 & 1,60 & 1,32 & 0,756 & 0,462 & \\
\hline R13 & 2,84 & 0,31 & 1,30 & 1,41 & 0,772 & 0,468 & \\
\hline R14 & 2,90 & 0,48 & 1,24 & 1,86 & 0,704 & 0,591 & \\
\hline R15 & 1,04 & 0,57 & 1,22 & 1,59 & 0,714 & & 0,493 \\
\hline R16 & 1,05 & 0,26 & 1,15 & 1,76 & 0,726 & & 0,491 \\
\hline R17 & 1,92 & 0,83 & 1,39 & 1,77 & 0,701 & & 0,384 \\
\hline R18 & 1,06 & 0,93 & 1,47 & 1,44 & 0,735 & & 0,412 \\
\hline R19 & 1,07 & 0,99 & 1,65 & 1,34 & 0,794 & & 0,485 \\
\hline R20 & 1,01 & 0,80 & 1,47 & 1,45 & 0,752 & & 0,384 \\
\hline R21 & 1,82 & 0,74 & 1,20 & 1,66 & 0,734 & & 0,461 \\
\hline R22 & 1,16 & 0,69 & 1,49 & 1,46 & 0,705 & & 0,401 \\
\hline R23 & 1,19 & 0,36 & 1,57 & 1,50 & 0,772 & & 0,493 \\
\hline R24 & 1,06 & 0,46 & 1,67 & 1,68 & 0,785 & & 0,524 \\
\hline R25 & 1,01 & 0,51 & 1,32 & 1,50 & 0,705 & & 0,395 \\
\hline R26 & 1,07 & 0,55 & 1,32 & 1,59 & 0,715 & & 0,330 \\
\hline & & & & & & & \\
\hline
\end{tabular}

$\mathrm{R}=$ Reactivo, $\mathrm{M}=$ Media, $\mathrm{D}=$ Desviación Estándar, $\mathrm{S}=$ Sesgos, $\mathrm{C}=$ Curtosis, $\mathrm{A}=$ Alfa quitando el valor del item. BROOTSTRAP $=0,000$; CURTOSIS $=1,304 ; \mathrm{KMO}=0,721 ;$ BARTLETT $\left[\chi^{2}=18,33(18 \mathrm{gl}) \mathrm{p}=\right.$ $0,000], \mathrm{F} 1$ - Actitud hacia la identidad de grupos cercanos a portadores de VIH /SIDA ( $43 \%$ de la varianza total explicada), F2 = Actitud hacia el estigma de grupos cercanos a portadores de VIH $/$ SIDA ( $57 \%$ de la varianza explicada)

Fuente: Elaborada con los datos del estudio

García (2018) en donde reportaron valores alfa de confiabilidad superior a , 800 .

Carreón, Hernández, Bustos y García (2018) propusieron un instrumento más específico que midió el compromiso laboral de los profesionistas de la salud con respecto a sus funciones preventivas y de atención social, encontrando un alto compromiso para quienes contaban con bajos ingresos en relación con quienes poseen los recursos financieros para su atención privada.

En el presente trabajo la dimensión de estigma abarca las dimensiones establecidas en el compromiso laboral, pero los valores alfa de consistencia interna parecen sugerir una deseabilidad social que atañe a servidores públicos y profesionistas de la salud incluso en su misma elección de 
carrera, desempeño académico y practica laboral (García, Bustos, \& Carreón, 2017).

Por consiguiente, es menester la aplicación de una escala de deseabilidad social para indagar el sesgo académico y formativo con el que los profesionistas de la salud llevan a cabo su desempeño.

\section{Conclusión}

El objetivo del presente trabajo fue establecer la confiabilidad y la validez e un instrumento que mide la actitud hacia grupos cercanos a portadores de VIH / SIDA, pero el tipo de estudio no experimental, el tipo de muestreo no probabilístico y el tipo de análisis exploratorio limitaron los resultados al contexto de la investigación, sugiriendo la aplicación de un instrumento de deseabilidad social.

Figura 2. Modelo estructural de trayectorias de dependencia y reflejantes

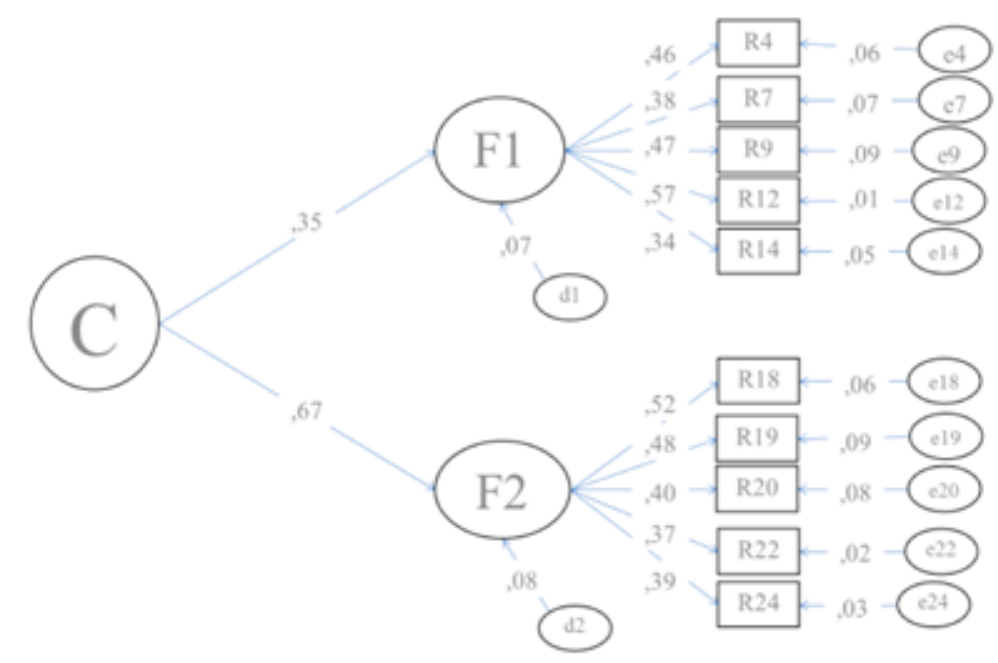

$\mathrm{C}=$ Actitud hacia grupos cercanos a portadores del VIH $/$ SIDA, F1 $=$ Actitudes hacia la identidad de grupos cercanos a portadores de VIH / SIDA, F2 = Actitud hacia el estigma de grupos cercanos a portadores de VIH $/$ SIDA: $\mathrm{R}=$ Reactivo, $\mathrm{d}=$ Disturbio, $\mathrm{e}=$ Error de medición 


\section{Referencias}

Abbasi, A., Rafique, M., Aziz, W., \& Hussai, W. (2013). Human immunodeficiency virus / acquired immune deficiency syndrome (HIV/AIDS): Knowledge, attitudes of university students of the state of Azad Kashmir (Pakistan). Journal of AIDS \& HIV Research, 5, 157-162.

Afanador, A. (2014). Particularidades con respecto a la formación de la sexualidad en los adolescentes. Cuadernos Hispanoamericanos de Psicología, 13(2), 91-104.

Albacerrin, D. \& Wyer, R. (2011). Elaborative and non elaborativeproccess a behaviorrelatedcommunication. Personality and Social PsychologyBulleting, 27, 691-705.

Aramayo, S. (2011). Terapia centrada en las soluciones aplicadas a la agresión sexual. Estudio de caso. Revista Ajayu, 9, 132-168.

Bautista, M., Delgado, M. A., García, C., Valdés, O., Hernández, G Castro, A., \& Trujillo, K. V. (2016). Contrastación de un modelo de cultura organizacional en centros de salud comunitaria ante la difusión informativa del VIH/SIDA desde e trabajo social. Enseñanza e Investigación en Psicología, 21(3), 248-255.

Becerra, V., Chunga, N., Palomino, C., Arévalo, T., Nivin, J., Portocarrero, L., Carbajal, P., Tomás, B., Caro, M., Astocaza, L., Torres, L., Carbajal, E., Pinto, A., Moras, M., Munayco, M., \& Gutiérrez, C. (2012). Asociación entre conocimiento de mujeres peruanas hacia VIH y sus actitudes frente a personas infectadas. Revista Peruana de Epidemiología, 16, 1-8.

Berenson, K., Paprocky, C., Fishman, M. T., Bhusman, D., ElBassel, N., \& Downey, G. (2015). Rejection sensitivity, perceived power, and HIV risk in the relationships of low income urban women. Women \& Heatlh, 55(8), 900-920.

Cañizo, E. \& Salinas, F. (2010). Conductas sexuales alternas y permisividad en jóvenes universitarios. Enseñanza e Investigación en Psicología. 15, 285-309.

Carreón, J., Bustos, J. M., Hernández, J., Quintero, M. L., \& García, C. (2015). Confiabilidad y validez de un instrumento que mide actitud hacia grupos cercanos a portadores del VIH / SIDA. Eureka, 12(2), 218-230.

Carreón, J., Hernández, J., Bustos, J. M., \& García, C. (2018). Confiabilidad y validez de un instrumento que mide el compromiso laboral de trabajadoras sociales en instituciones de asistencia sanitaria. Encrucijada, 26, 69-86.

Castillo, A. \& Chinchilla, Y. (2011). La experiencia de la escuela de psicología de la Universidad de Costa Rica en la atención de la explotación sexual comercial. Revista Latinoamericana de Derechos Humanos, 22, 121-151.

Chacón, M., Barrantes, K., Comerfold, M., \& McCoy, C. (2014). Prácticas sexuales y conocimiento sobre el VIH/SIDA entre usuarios de drogas en una comunidad de bajos ingresos en Costa Rica. Salud y Drogas, 14(1), 27-36.

Davis, M., Shell, A., \& King, S. (2012). Assessing pharmacist' perspectives of HIV and the care of HIV-infected patients in Alabama. Pharmacy Practice, 10, 188-193.

Ferragut, M. \& Ortiz, M. (2013). Psychological values as protective factors against sexist attitudes in preadolescents. Psicothema, 25, 38-52.

García, C. (2013). Actitud de trabajadoras sociales hacia portadores del Virus de Inmunodeficiencia Humana en centros de salud comunitaria. Salud \& Sociedad, 4(1), 60-68.
García, C., Bustos, J. M., \& Carreón, J. (2017). Dimensiones exploratorias del estigma social y laboral. Un estudio Delphi con estudiantes de pregrado. Eureka, 14(1), 55-68.

García, C., Carreón, J., Sandoval, F. R., Bustos, J. M., \& Aguilar, F. (2016). Estructura de la cultura laboral en una institución de promoción de salud pública. Arequipa, 6(1), 291-304.

García, I. \& Rodríguez, M. (2014). Situación en que viven y adhesión al tratamiento en mujeres y jóvenes de San Luis Potosî con VIH/SIDA. Acta Universitaria, 24(4), 3-14.

Giraldo, I. (2013). Cibercuerpos: los jóvenes y sexualidad en la posmodernidad. Actualidades Investigativas en Educación, $13,1-22$.

Guerra, V. M. \& Gouveia, V. V. (2007). Liberalismo / conservadurismo sexual: proposta de uma medida factoirial. Psicología, Reflexao e Critica, 20(1), 43-53.

Hernández, I. (2013). Haciendo camino al andar: Migración, feminización y trata de personas en los flujos de migración irregular en la frontera sur de México. Revista Digital Universitaria, $14,1-15$.

Hughes, S. \& Barnes, D. (2011). The dominance of associative theorizing in implicit attitude research: propositional and behavioral alternatives. Psychological Research, 6, 465-498.

Hurtado, N., Avandaño, M., \& Moreno, F. (2013). Embarazo en la adolescencia: entre el fracaso informativo y el logro psíquico. Revista de Psicología de la Universidad de Antioquia, 5(1), 93-102.

Jouen, F. \& Zielinski, S. (2013). La explotación sexual comercial de menores en los destinos turísticos. Conocimientos, actitudes y prevención de los prestadores de servicios turísticos en Tananga, Colombia. Revista de Turismo y Patrimonio Cultural, 11, 121-134.

Klaus, A., Piñeres, J., \& Hincapie, A. (2010). Subjetivaciones lenguaje y parodia: reflexiones en torno a los discursos expertos sobre la explotación sexual comercial de niños y niñas adolescentes. Revista Latinoamericana de Ciencias Sociales, Niñez y Juventud, 8, 269-291.

Krimberg, B., Saldanha, M., \& Neves, M. (2014). Mulheres e on HIV / AIDS: Interseccoes entre genero, feminism, psicologia e salud publica. Revista Colombiana de Psicología, 23(2),285-296.

Lloret, S., Ferreses, A., Hernández, A., \& Thomas, I. (2014). El análisis factorial exploratorio de los ítems: Una guía práctica, revisada y actualizada. Anales de Psicología, 30(33), 1151-1169.

Mannell, J., Cornish, F., \& Russell, J. (2014). Evaluating social outcomes of HIV/AIDS interventions: a critical assessment of contemporary indicators frameworks. Journal of International AIDS society, 17(1), 1-11. doi https://doi.org/10.7448/IAS.17.1.19073

Mardones, R. \& Guzmán, M. (2011). Hacia un tratamiento integral desde el modelo psicosocial en niños, niñas y adolescentes explotados sexualmente. Revista Científica de Psicología, 2, 27-47.

Méndez, M. (2013). Trata: esclavitud invisibilizada en Costa Rica. Estudio de cinco casos. Revista Costarricense de Psicología, 32, 109-135.

Méndez, R., Rojas, M., \& Moreno, D. (2012). Explotación sexual comercial infantil: las rutas de la vida del maltrato. Investigación y Desarrollo, 20, 450-47.

Meneses, A., Avellar, T. H., Veloso, V., Nunes, P., \& Pinheiro, B. L. (2015). Noopsicosomaticas em pessoas viviendo com HIV / AIDS: Evidencias de um modelo explicativo. Psico, 46(1), 129-138. 
Orcasita, L. T., Cuenca, J., Garrido, D., \& Haderlein, A. (2018). Diálogos y saberes sobre sexualidad de padres con hijos e hijas adolescentes escolarizados. Revista Colombiana de Psicología, $27,41-53$.

Orcasita, L. T., Uribe, A. F., \& Valderrama, L. J. (2012). Conocimientos y actitudes frente al VIH / SIDA en pares de familia de adolescentes colombianos. Revista Colombiana de Psicología, 22 (1), 59-73.

Petro, B. (2013). Attitudes and views of teachers towards student sexual relationships in secondary school in Tanzania. Academic Research International, 4, 232-241.

Rivers, M. (2011). Los usos de la trata en Centroamérica: migración, género y sexualidad. Anuario de Estudios Centroamericanos, 37, 87-103.

Rodríguez, D. (2013). Enfermedad crónica avanzada, padecimiento psíquico y sistema general de la seguridad social en la salud. Revista de Psicología de la Universidad de Antioquia, 5(1), 75-92.

Selesho, J. \& Modise, A. (2012). Strategy (ies) in daling with HIV / AIDS in our schools: changing the lenses. Journal of Human Ecology, 38, 181-189.
Solat, S., Velhal, G., Mahajan, H., Rao, A., \& Sharma, B. (2012). Assesment of knowledge and attitude of rural population about HIV/AIDS in Raigad Distrit, India. Journal of Dental and Medical Science, 1, 31-45.

Summer, L. (2011). The theory of planned behavior and the impact of the past behavior. International Business and Economic Research Journal, 10, 91-110.

Uribe, A. F. (2005). Intervención grupal y calidad de vida en personas diagnosticadas de VIH. Revista Colombiana de Psicología, 14, 28-33.

Uribe, A. \& Orcasita, L. (2011). Evaluación de conocimientos, actitudes, susceptibilidad y autoeficacia frente al VIH / SIDA en profesionales de salud. Avances de Enfermería, 29, 271-284.

Villa, E. (2010). Estudio antropológico en torno a la prostitución. Cuicuilco, 17, 157-179.

Villegas, E., Rosas, F. J., \& García, C. (2018). Social Works towards a quality life and wellbeing subjective. International Journal of Research in Humanities and Social Studies, 5(6), 35-48. 